\title{
A Robust Visual Tracking of an Arbitrary-Shaped Object by a New Active Contour Method for a Virtual Reality Application
}

\author{
Cheng-Ming Huang ${ }^{1}$, Su-Chiun Wang ${ }^{1}$, Li-Chen Fu ${ }^{1,2}$, Pei-Ying Chen ${ }^{1}$, Yu-Shan Cheng ${ }^{1}$ \\ Department of Electrical Engineering ${ }^{1}$ \\ Department of Computer Science and Information Engineering ${ }^{2}$ \\ National Taiwan University, Taipei, Taiwan, R.O.C. \\ E-mail: lichen@ccms.ntu.edu.tw
}

\begin{abstract}
This paper presents a real-time, highly reliable, open field visual tracking system, which can automatically detect an arbitrary-shaped object tin 3-D space and find out its location so that the 2-DOF camera platform can be controlled to keep the target centered in the monitor image. The total processing period of the proposed visual servo system is less than $34 \mathrm{~ms}$. Next, the visual servo system is to play the role as a military light-of-sight on the air fighter to lock an enemy fighter. Incidentally, the 2-DOF information of the camera platform is adopted the autopilot control of an air fighter to track the foregoing target. The overall system is experimented and verified in a virtual reality environment, which can further be developed as an entertainment or military training platform.
\end{abstract}

Keywords: Visual tracking, visual servo, virtual reality.

\section{Introduction}

Visual tracking has been an important topic in computer vision and robotics fields. For practical visual tracking systems, there are some basic functionalities required: real-time, automatic, and robustness in non-ideal situations, such as occlusions and cluttered environment. Surveys show that individual problems mentioned above have been solved. For example, CONDENSATION algorithm [5] is highly regarded since it solved both real-time and robustness problem, but not an automatic one. Up to now, however, no literature seems to be found able to cope with all the problems completely. Therefore, an integrated algorithm is presented in this paper to overcome the overall problems by combining template matching [10-12] and Snake-based counter matching [1-3, 13-14]. The proposed algorithm also improves the disadvantages of classical Snake [1-3]. On the other hand, virtual reality (VR) is a kind of advanced technology which facilitates scientists and engineers to simulate and to verify their works in an interactive application environment. Apparently, unmanned air vehicle (UAV) experiments pose really challenging environments for various related conduct researches [9]. Owing to this condition, before performing that real experiment, this paper proposes a multi-plane simulator which integrates the visual tracking algorithm and the VR presentation technique so that a UAV equipped with a visual tracker can be tested in lab.

\section{Visual Tracking System}

The architecture of our visual tracking system can be easily shown by the block diagram in Fig.1. It consists of four subsystems, including motion detection, Snake-based outline extraction, hybrid tracking algorithm and visual probability data association (VPDA) filter. The on-line image sequences are grabbed by a camera, which is mounted on a pan-tilt servo platform.

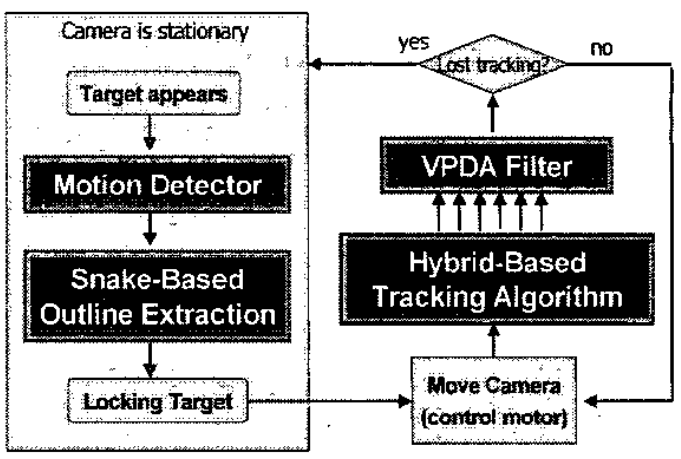

Fig.1. The architecture of visual tracking system.

\subsection{Motion Detection}

There are several advantages to use motion detection 
before tracking an object. First, it provides important clues to an automatically tracking system. Second, we can determine a smaller searching range to reduce the computational time of finding out the position of the moving object in the image, obtained by motion detection with a stationary camera. Third, the segmentation of the moving object can be the basis of automatic initialization of Snake.

As shown in Fig. 2, two consecutive image frames $I(k)$ and $I(k-1)$ are subtracted pixel by pixel, and the results are then binarized. If the total number of white pixels is less than the moving threshold $\mathrm{Thr}_{\text {moving, the }}$ motion detection unit continues to examine the next two consecutive frames. On the contrary, if the number exceeds the moving threshold, it means a target to be detected. The concept of "moving edge" is included by doing a logic AND operation between the subtracted image and the edge image of the current frame. We can yield a more accurate position of the target outline from the moving edge image. Finally, the moving edge image is submitted to generate a proper-sized initialization of Snake for outline extraction followed by tracking algorithm.

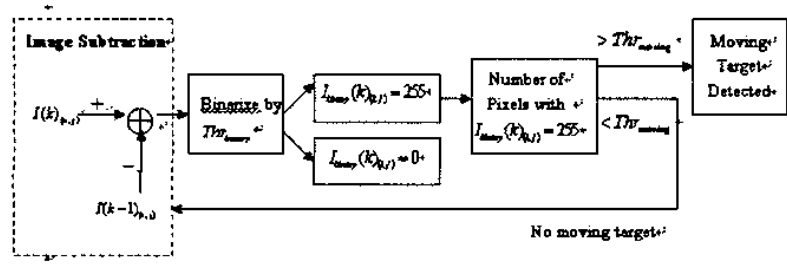

Fig. 2. Block diagram of the motion detection.

\subsection{Snake-Based Outline Extraction}

The objective of the section is to extract the outline of the target by active contour models (also called Snakes) [1]. A modified Snake is presented here on the basis of the Greedy algorithm [2]. The proposed Snake has an external constraint force to speed up the convergence to the desired feature of the object, and provides an easy way to avoid the wrong convergence on a noise point. If $v_{i}=\left(x_{i}, y_{i}\right)$ for $i=0,1, \ldots, N$ represents the $N$-length discrete contour, the modified Snake energy is

$$
\begin{aligned}
E_{\text {smaks }}^{*} & =\sum_{i=1}^{N} E_{\text {snake }}\left(v_{i}\right) \\
& =\sum_{i=1}^{N}\left\{\alpha_{i} E_{\text {cont }}\left(v_{i}\right)+\beta_{i} E_{c u n}\left(v_{i}\right)+\gamma_{i} E_{i}\left(v_{i}\right)+\eta_{i} E_{\text {dis }}\right\}
\end{aligned}
$$

where $E_{c o n t}$ is the continuity energy, $E_{c u r v}$ is curvature energy, $E_{I}$ is the image energy and $E_{d i s}$ is the constraint energy, called distance energy. The parameters $\alpha_{i}, \beta_{i}, \gamma_{i}$ and $\eta_{i}$ are used to balance the relative influence of the four terms. The mathematical formula of each energy term is

$$
\begin{aligned}
& E_{\text {cont }}=\left|\frac{\bar{d}-\left|V_{i}-V_{i-1}\right|}{M A X\left\{\bar{d}-\left|V_{i}(j)-V_{i-1}\right|\right\} \mid}\right| \\
& E_{c u r v}=\frac{\left|V_{i-1}-2 V_{i}+V_{i+1}\right|^{2}}{M A X\left\{\left|V_{i-1}-2 V_{i}(j)+V_{i+1}\right|^{2}\right\}}, \\
& E_{l}=\frac{\nabla I_{\max }-\nabla I\left(v_{i}\right)}{\nabla I_{\max }-\nabla I_{\min }} \\
& E_{\text {dis }}=\frac{D_{\max }-D\left(v_{i}\right)}{D_{\max }-D_{\min }}
\end{aligned}
$$

where $\bar{d}$ is the average distance between contour points and $V_{i}(j)$ represents the eight neighbors of a point $V_{i}$ for $j=1, \ldots, 8$. Moreover, $\nabla I\left(v_{i}\right)$ denotes the image intensity of the edge image at current position, $D\left(v_{i}\right)$ denotes the absolute value of the distance between the current position and the center of the object, and $\nabla I_{\max }\left(D_{\max }\right)$ and $\nabla I_{\min }\left(D_{\min }\right)$ denote the maximum and minimum image intensity (distance from the center of the object) in the neighborhood, respectively. The moving direction of the Snake can be decided by adding or subtracting the distance energy $E_{\text {dis }}$. Hence, the automatic initialization of Snake can be solved by combining the modified Snake and motion detector, which will be described in next section.

The concept of Greedy algorithm [2] is to take minimized $E^{*}$ snake as sum of each single minimized $E_{\text {snake }}\left(v_{i}\right)$, that is

$$
\min E_{\text {snake }}^{*} \approx \sum_{i=1}^{N} \min E_{\text {snake }}\left(v_{i}\right) .
$$

Figure 3(a) demonstrates how the iterative Greedy algorithm works. The energy function is computed for the current position $v_{i}$ and each of its neighbors. Then, the location having the smallest energy value is chosen as the new position $v_{i}{ }^{\prime}$. As a result of repeating the aforementioned process point by point, all points of the contour keep moving forward to their corresponding new positions to form a new contour. Throughout the processes, 
the Snake completes an iteration of the deformation loop. The Snake will repeat the deformation loop iteratively until it converges to the desired feature of the object, as shown in Fig. 3(b).

From the result of motion detector, a moving object is detected. Assume the whole body of the object is completely inside a $140 \times 140$ area, which is the searching area. We choose a proper-sized ellipse (including circles), which can enclose the whole moving object inside, as an initialization of Snakes. The center of ellipse is located at the center of the bounding box that contains the object inside. Then, axes length of the initial contour are determined according to the width and height of the bounding box. At the same time, we also use the center of the minimized rectangle as that of the searching area, as illustrated in Fig. 4.

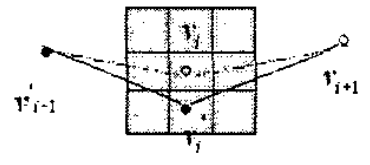

(a)

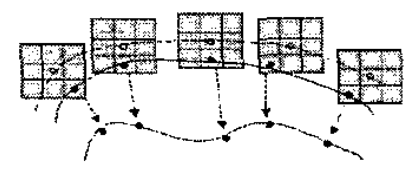

(b)
Fig. 3. (a) The demonstration of Greedy algorithm. (b) The deformable of Snake. (a)

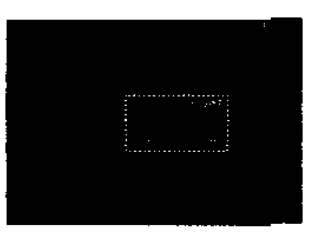

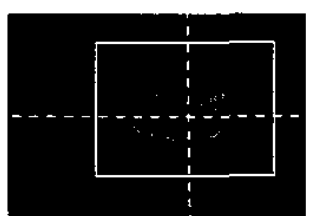

(b)
Fig. 4. In moving edge image, (a) shows the bounding box of the object, and (b) is the searching area and its center.

\subsection{Hybrid Tracking Algorithm}

Different from the traditional Snake-based tracking algorithms [3-10], Snake is only used to extract the outline of the target rather than to perform tracking in our system. Since Snake-based tracking algorithms should be under the assumption of slowly moving object, which unfortunately imposes serious constraints on the general use of tracking. This paper presents a contour matching method which makes use of the extracted contour model for tracking an arbitrary- shaped object. But the method also has a restriction against low-contrast environment, just like what the Snake-based tracking algorithms encountered. Hence, we integrate the most commonly used method in visual tracking, called template matching, with contour matching to overcome the foregoing drawback.
The details of contour matching are described as follows. To highlight the contour of an object in an image, the edge image is used for object tracking (see Fig.5). Similar to template matching, we need to sum the total gradient values pixel by pixel in the edge image along a pre-extracted contour model. After summing over a contour model, we will shift the center of contour model to the next pixel and compute the total sum along the perimeter of the contour model again. After going through all the searching area, we can get the largest summation corresponding to the distribution of edge pixels which resembles the object's contour. The numerical process in each searching loop mentioned above can be summarized by the following normalized sum equation

$$
\phi_{g}(s)=\frac{1}{N_{\sigma}} \sum_{i=1}^{N_{\sigma}}\left|g_{i}\right| \text {, }
$$

where $s=(x, y)$ represents the position of the center of contour model, $N_{\sigma}$ is the total number of all pixels along the perimeter of contour model, and $g_{i}$ denotes the gradient value of each pixel along the perimeter of contour model. Combined with SAD template matching, the best object position is

$$
\mathbf{s}^{*}=\arg \max _{\mathbf{s}_{i} \in S}\left\{\bar{\phi}_{g}\left(\mathbf{s}_{i}\right)+\bar{\phi}_{S A D}\left(\mathbf{s}_{i}\right)\right\},
$$

where

$$
\begin{aligned}
& \bar{\phi}_{g}(\mathbf{s})=\frac{\phi_{g}(\mathbf{s})-\min _{\mathbf{s}_{i} \in S} \phi_{g}\left(\mathbf{s}_{i}\right)}{\max _{\mathbf{s}_{i} \in S} \phi_{g}\left(\mathbf{s}_{i}\right)-\min _{\mathbf{s}_{i} \in S} \phi_{g}\left(\mathbf{s}_{i}\right)} \text { and } \\
& \bar{\phi}_{S A D}(\mathbf{s})=\frac{\max _{\mathbf{s}_{i} \in S} \phi_{S A D}\left(\mathbf{s}_{i}\right)-\phi_{S A D}(\mathbf{s})}{\max _{\mathbf{s}_{i} \in S} \phi_{S A D}\left(\mathbf{s}_{i}\right)-\min _{\mathbf{s}_{i} \in S} \phi_{S A D}\left(\mathbf{s}_{i}\right)}
\end{aligned}
$$

are the normalized forms, in which $\mathbf{S}$ is the center position of the contour model as well as the template, and $S$ is the searching area.

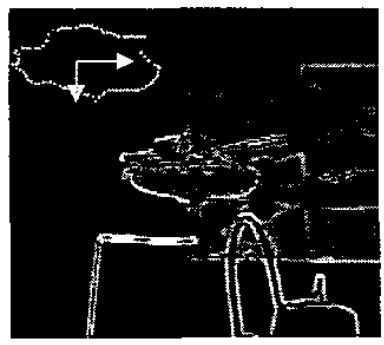

Fig. 5. Search the object by contour matching in the edge image. 


\section{Trajectory Design}

Highly cluttered environment may contain many background contents that are quite similar to the target and then result in false alarms, which may cause further error accumulation in the subsequent tracking. Not only a bad template or contour will be involved, but also the trajectory detection will be mislead and seriously affect the position of the searching window. Thus, it gives rise to a high possibility of lost of tracking once the best match is a false match. For that reason, Visual Probabilistic Data Association (VPDA) is adopted to overcome the challenge in the cluttered environment. It can provide a more reliable approach to predict the next position of the target, and enhance the robustness of the tracking system, even in cluttered environment. Actually, the VPDA filter is the original probabilistic data association (PDA) filter [7] integrated with the visual information, introduced in [8].

The concept of the PDA filter is to take all possible targets into account instead of only the best matched one that may be correspond to a part of the cluttered environment, and then produce a weighted- average output from all possible candidates. The method behind is the probability technique. It computes the posterior association probabilities for all current possible candidate measurements and uses them to form a weighted sum of innovations for updating the target's state in a suitably modified version of the Kalman Filter.

\section{A Virtual Reality Application}

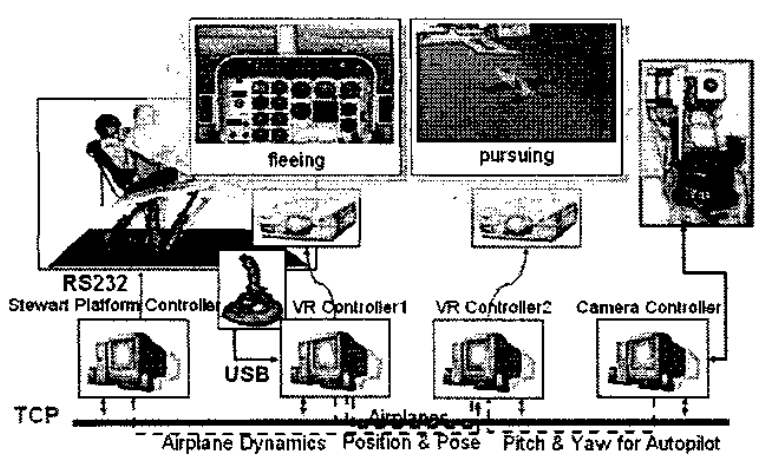

Fig. 6. The architecture of the overall system.

As has been mentioned in introduction, the hereby proposed visual servo algorithm is taken to build a virtual reality system involving UAV which allows simulation and evaluation of the control performance of some UAV equipped with a visual tracker. As shown in Fig. 6, there are two air fighters in the virtual reality environment. The fleeing one is operated through force-feedback joystick by the pilot who sits on a steward platform. The pursuing one is mounted with the visual tracking system as mentioned above. We want to emphasize that the visual tracking system here is to play the role as a military light-of-sight to lock on the target. Also, we can use the pan and tilt information of the camera to aid the autopilot of the pursuing air fighter. In other words, the visual tracker of the pursuer in the virtual world will be replaced by the camera mounted on a motion platform, and the VR image will be presented from the viewpoint of the pursuer so that the visual servo system can successfully fulfill its endowed function. Each subsystem communicates with one another with TCP/IP protocol.

\section{Experimental Results}

The performance of the Snake-based outline extraction is quite appealing and very fast even if the object appears in highly cluttered environment, as shown in Fig. 7. Figure 8 and 9 demonstrate tracking of a moving tank model and a VR target, respectively. In the VR autopilot case, we can see that the fleeing one is chased by the pursuer and is kept at the center of the screen, as shown in Fig. 10, from the viewpoint of the pursuer. Experimental results show that our tracking system is highly robust against occlusion, low-contrast environment, and rapid motion.

\section{Conclusions}

The major contribution of this work is to establish an integrated visual servo system, which can track an arbitrary-shaped object in a highly noisy environment, even with some occlusions on the target. The visual servo system can perform the tracking processes with $320 \times 240$ image size in nearly real-time constraint (less than $34 \mathrm{~ms}$ ) and center the target up to 15-pixel square range tolerance. We also make several improvements from Snake and reenhance the visual tracking method with a hybrid tracking method and VPDA filter. Furthermore, we propose a new application in this paper which simulates and verifies the visual servo system in the virtual reality environment. In the future, we will combine the Stewart platform and the force-feedback joystick as shown in Fig. 6 to build an entertainment or military training system.

\section{References}

[1] M. Kass, A. Witkin, and D. Terzopoulos, "Snake: active contour models," Int. J. Comput. Vis., Vol. 1, pp. 321-331, 1987.

[2] D.J. Williams and M. Shah. "A fast algorithm for active contours and curvature estimation," CVGIP: Image 
Understanding, Vol. 55, No. 1, pp. 14-26, Jan. 1992.

[3] C. Xu and J.L.Prince, "Snakes, shapes, and gradient vector flow", IEEE Trans. Image Processing, Vol. 7, No. 3, pp. 359-369, 1998.

[4] J. Denzeler and H. Niemann, "A two stage real-time object tracking system," In Pavesi'c et al.

[5] M. Isard and A. Blake, "CONDENSATIONconditional density propagation for visual tracking," Int. $J$. Computer Vision, pp. 1-36, 1998.

[6] J. Denzeler and H. Niemann, "A new energy term combining Kalman filter and active contour models for object tracking," Machine Graphics Vision, Vol. 5(1/2), pp. 157-165, 1996.

[7] Y. B. Shalom and E. Tse, "Tracking in a cluttered environment with probabilistic data association," Automatica, Vol. 11, pp. 451-460, 1975.

[8] D. Liu, "Real-time visual tracking in cluttered environment with a pan-tilt camera," Master Thesis, Dept. of E.E., National Taiwan University.
[9] A. H. Göktoğan, E. Nettleton, M. Ridley, and S. Sukkarieh, "Real time multi-UAV simulator," Proc. IEEE ICRA, pp. 2720-2727, 2003.

[10] R. Brunelli and T. Poggio, "Template matching: matched spatial filters and beyond," MIT AI Memo 1549, July 1995 .

[11] M. S. Lew, N. Sube, and T. S. Huang, "Improving visual matching," IEEE Conference on Computer Vision and Pattern Recognition, Vol. 2, pp. 58-65, 2000.

[12] Y. S. Chen, Y. P. Hung, and C. S. Fuh, "A fast block matching algorithm based on the Winner-update strategy," Proc. of the 4th Asian Conf. on Computer Vision, Vol. 2, pp. 977-982, 2000.

[13] S. Birchfield, "An elliptical head tracker," $31^{\text {st }}$ Asilomar Conference on Signal, Systems, and Computers, November 1997.

[14] S. Birchfield, "Elliptical head tracking using intensity gradients and color histograms," IEEE Conf. on Computer Vision and Pattern Recognition, Santa Barbara, California, July 1998.

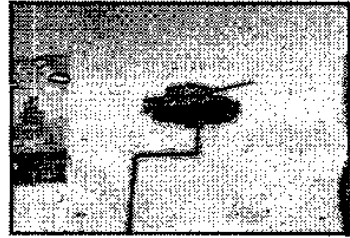

(a) current image

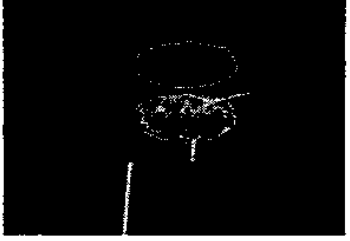

(b) initial contour

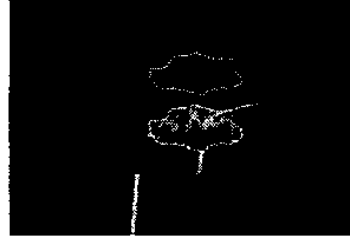

(c) extracted outline

Fig. 7. It takes only 9 iterations to extract the tank's outline with 84 pixel-length of initial contour.
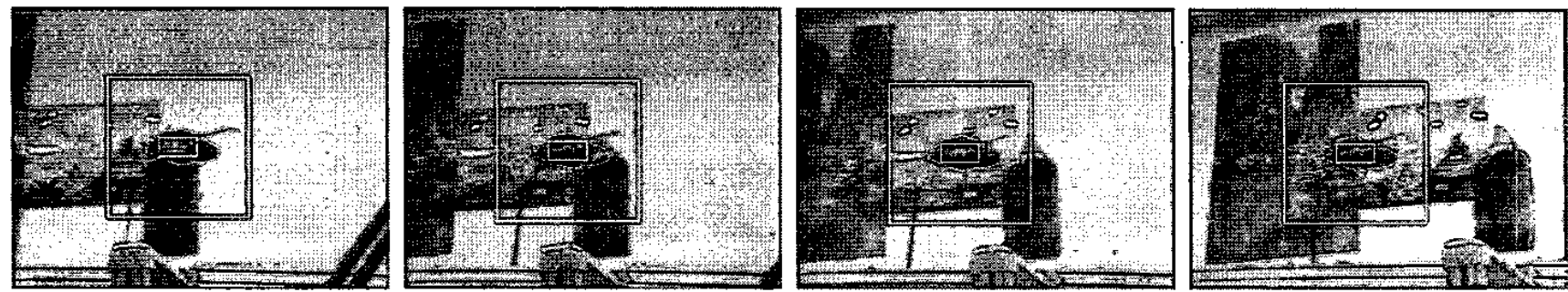

Fig. 8. Tracking tank model in the cluttered environment with occlusions. 

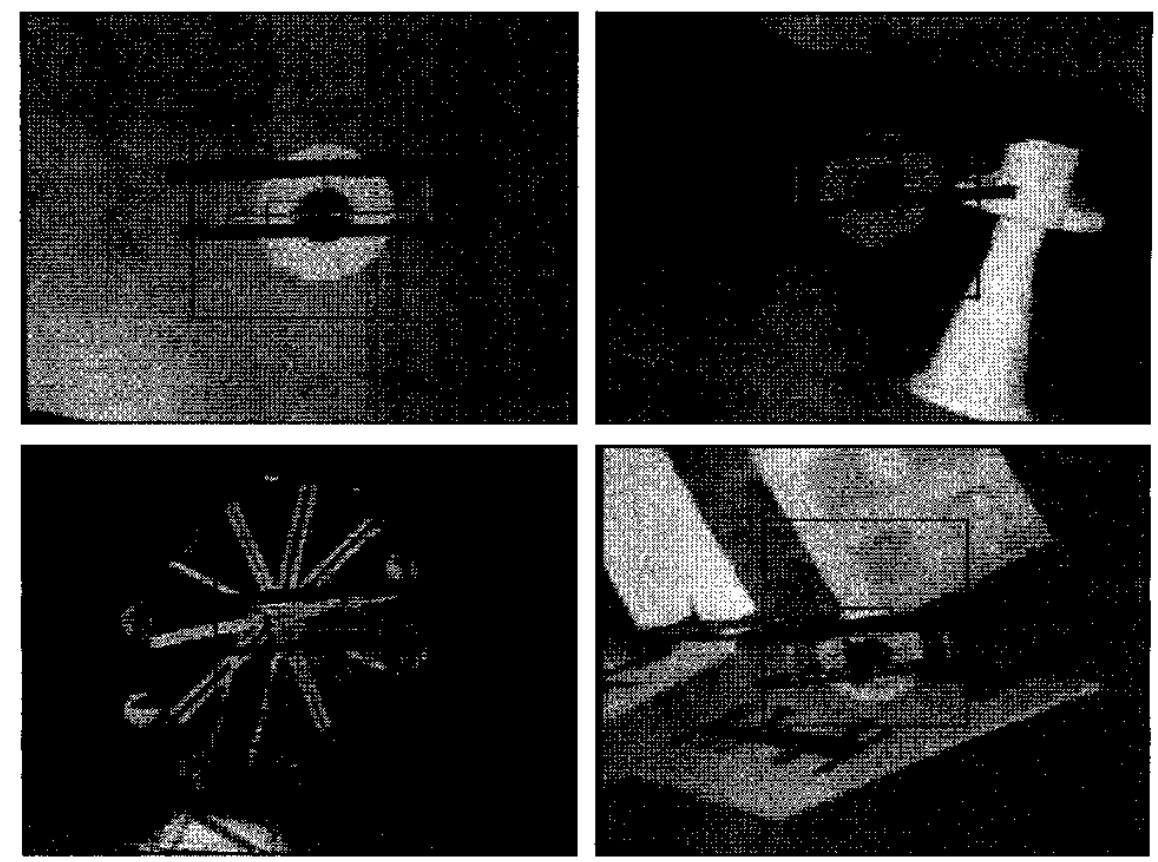

Fig. 9. Tracking the air fighter in the various VR cases.
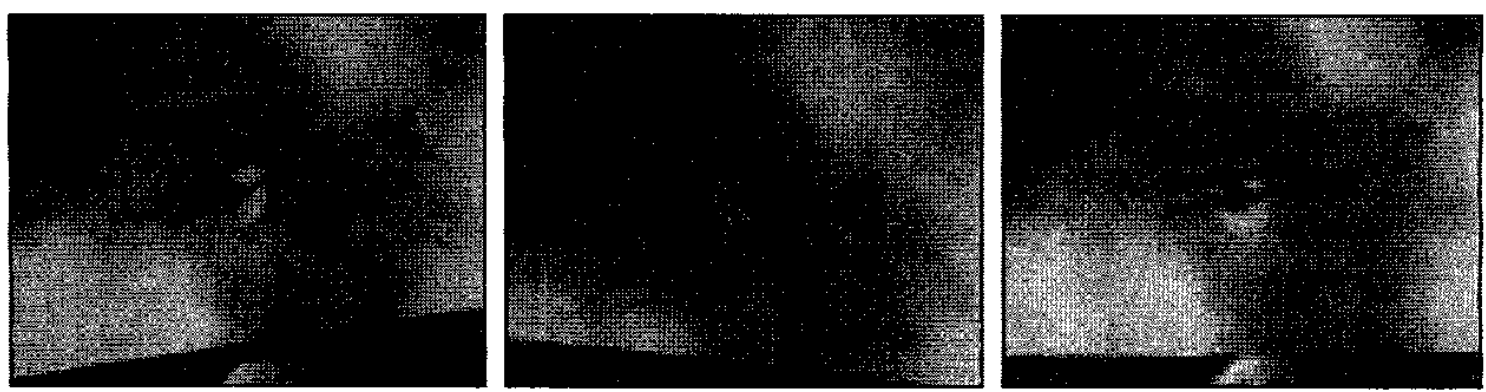

Fig. 10. The autopilot ability shown from the viewpoint of the pursuer. 